\title{
Fenofibrate attenuates contact-stimulated cell motility and gap junctional coupling in DU-145 human prostate cancer cell populations
}

\author{
EWA WYBIERALSKA ${ }^{1}$, KATARZYNA SZPAK ${ }^{1}$, ANDRZEJ GÓRECKI ${ }^{2}$, PIOTR BONAREK ${ }^{2}$, \\ KATARZYNA MIĘKUS ${ }^{3}$, JUSTYNA DRUKAŁA ${ }^{1}$, MARCIN MAJKA $^{3}$, KRZYSZTOF REISS ${ }^{4}$, \\ ZBIGNIEW MADEJA ${ }^{1}$ and JAROSŁAW CZYŻ
}

\begin{abstract}
Departments of ${ }^{1}$ Cell Biology and ${ }^{2}$ Physical Biochemistry, Faculty of Biochemistry, Biophysics and Biotechnology, Jagiellonian University, Cracow; ${ }^{3}$ Department of Transplantation, Polish-American Institute of Pediatrics, Jagiellonian University School of Medicine, Cracow, Poland; ${ }^{4}$ Neurological Cancer Research, Stanley S. Scott Cancer Center, Louisiana State University Health Sciences Center, New Orleans, LA, USA
\end{abstract}

Received March 9, 2011; Accepted April 14, 2011

DOI: $10.3892 /$ or.2011.1321

\begin{abstract}
In the present study, we investigated the effects of fenofibrate on the invasive potential of DU-145 human prostate cancer cells in the context of gap junctional intercellular coupling and the formation of reactive oxygen species. Timelapse analyses of cell motility, accompanied by tests of cell viability, membrane microviscosity, reactive oxygen species accumulation and the function of gap junctional protein connexin 43 were performed in monolayer cultures of DU-145 cells following fenofibrate administration. Fenofibrate inhibited the motility of DU-145 cells and attenuated gap junctional intercellular coupling in a manner independent of its effects on cell viability, PPAR $\alpha$ activation and cell membrane microviscosity. Instead, N-acetyl-L-cysteine, a scavenger of reactive oxygen species, restored cell motility and gap junctional coupling in fenofibrate-treated DU-145 cell populations. These data indicate that two parameters crucial for cancer cell metastatic potential, i.e. cell motility and gap junctional coupling, are inhibited by fenofibrate. Thus, fenofibrate affects prostate cancer cell invasion via an orchestrated action on versatile cancer cell properties determining this process. A novel mechanism of anti-invasive activity of fenofibrate, which depends on its interference with cell motility and the function of gap junctions regulated by reactive oxygen species, is suggested.
\end{abstract}

Correspondence to: Dr Zbigniew Madeja or Dr Jarosław Czyż, Department of Cell Biology, Faculty of Biochemistry, Biophysics and Biotechnology, Jagiellonian University, ul. Gronostajowa 7, Cracow 30-387, Poland

E-mail: z.madeja@uj.edu.pl

E-mail: jarek.czyz@uj.edu.pl

Key words: fenofibrate, motility, connexin, cancer, invasion, reactive oxygen species

\section{Introduction}

Active cell migration determining the capability of cancer cells to transmigrate natural barriers such as the endothelial continua and basement membrane is a key factor during the formation of metastases (1). However, the capability of cancer cells to penetrate vascular walls, a crucial stage of cancer invasion depending on cancer cell motility, is a function involving other cell properties, such as the expression of connexins and gap junctional intercellular coupling (GJIC). In particular, deregulation of GJIC facilitates early carcinogenesis and the formation of a local hyperplasia (2-5). In contrast, restoration of GJIC between cancer and normal cells is crucial for malignant cell dissemination (6-8).

Thus, new pharmaceutical regiments aimed at reducing the metastatic activity of cancer cells should be developed which are based on substances affecting both cell motility and the function of connexins. Compounds of the fibrate family have originally been described as effective vasoactive and cholesterol-lowering drugs. Among them, fenofibrate is commonly used in the prevention of arteriosclerosis as a lipid-lowering drug (9). It is of interest that fibrates in general exert a wide range of anti-cancer properties (10-14). In particular, fenofibrate was shown to interfere with the growth and survival of different cancer cell types in a manner dependent on the activation of peroxisome-proliferator activated receptor- $\alpha$ (PPAR $\alpha)$ (14-16).

The PPAR $\alpha$-dependent signaling pathway is regarded as a 'canonical' system which determines the effect of fenofibrate on cancer cell proliferation $(13,17)$. Moreover, the PPAR $\alpha$ dependent inhibitory effect of fenofibrate on cancer cell motility has been previously demonstrated (18). However, a PPAR $\alpha$-independent effect of fenofibrate on cell membrane rigidity was noted (19), while both its PPAR $\alpha$-dependent $(10,20)$ and PPAR $\alpha$-independent (21) effects on the accumulation of reactive oxygen species (ROS) were observed. Thus, the question arises concerning the possible involvement of ROS-dependent mechanisms of fenofibrate on cell 
properties which determine cancer progression, such as cell motility and GJIC.

'Contact-stimulated' cancer cell motility has long been regarded as a factor facilitating cancer cell invasion in general, and prostate cancer invasion in particular (22). The function of connexins and gap junctions in this process was recognized only recently $(8,23)$, but the influence of fenofibrate on cancer progression has not yet been discussed in the context of GJIC. However, the inhibitory effect of factors increasing ROS activity on the motility and function of gap junctions has been demonstrated (24). In the present study, we investigated the influence of fenofibrate on basic cellular parameters involved in prostate cancer invasion, such as cancer cell motility and gap junctional communication.

\section{Materials and methods}

Cell cultures. Human prostate carcinoma DU-145 cells were cultivated in DMEM-F12 HAM (Sigma, St. Louis, MO) medium supplemented with $10 \%$ FBS and antibiotics. For the endpoint tests, DU-145 cells were detached from the substratum with $0.25 \%$ trypsin in PBS and seeded in the culture wells at a density of 900 cells $/ \mathrm{mm}^{2}$. When indicated, basic medium was supplemented with fenofibrate, GW9662, and N-acetyl-L-cysteine (NAC; all from Sigma) at the concentration(s) indicated in the text.

Analyses of DU-145 cell motility and viability. DU-145 cell motility was time-lapse recorded at a density of 900 cells $/ \mathrm{mm}^{2}$ for $7 \mathrm{~h}$. The tracks of individual cells were determined from a series of changes in the cell centroid positions. The data were pooled and analyzed to elucidate basic cell motility parameters such as i) the total length of cell displacement (TLCD; $\mu \mathrm{m}$ ) and ii) the average velocity of cell movement (AVCM; $\mu \mathrm{m} / \mathrm{h}$ ) $(22,25)$. Cell trajectories from no less than three independent experiments (number of cells $>50$ ) were obtained for analysis by the non-parametric Mann-Whitney test. Independently, tests were performed at a cell density of 300 cells $/ \mathrm{mm}^{2}$ to elucidate the basic parameters of DU-145 cell morphology in the presence of various concentrations of fenofibrate. The length of cell periphery and the dispersion (defined as the minimum extension which is a measure of how much the cell shape differs from a circle) was analyzed $(26,27)$. To determine the effect of fenofibrate on cell viability, DU-145 cells incubated with different concentrations of fenofibrate at indicated times, were harvested, and the number of viable cells was determined by the fluorescence diacetate/ethidium bromide test.

Fluorescence anisotropy analyses. Membrane fractions of DU-145 cells cultured at a density of 900 cells $/ \mathrm{mm}^{2}$ in the medium supplemented with $100 \mu \mathrm{M}$ fenofibrate, $100 \mu \mathrm{M}$ cholesterol (positive control) and $5 \mathrm{mM}$ benzyl alcohol (negative control) for $2 \mathrm{~h}$ were harvested according to the established protocol (28). Fluorescence intensities were measured using a Fluorolog FL3-12 (Jobin Yvon) spectrofluorimeter equipped with a purpose designed polarizer at excitation and emission wavelengths of 350 and $426 \mathrm{~nm}$, respectively. In order to obtain a stability of the signal, the analysis was carried out for $30 \mathrm{~min}$ at 30 -sec time intervals (kinetics measurement). Mean values of the measured parameters are depicted as graphs.
FACS analyses of ROS generation by fenofibrate. DU-145 cells cultured at a density of 900 cells $/ \mathrm{mm}^{2}$ in DMEM-F12 HAM supplemented with $10 \%$ FBS for $24 \mathrm{~h}$ were treated with the medium containing $100 \mu \mathrm{M}$ fenofibrate or $100 \mu \mathrm{M}$ perhydrol (positive control) in the presence of fluorescence ROS indicator DHR 123 (dihydorhodamine 123; 1:500, Sigma) for $4 \mathrm{~h}$. Measurements of DHR 123 fluorescence

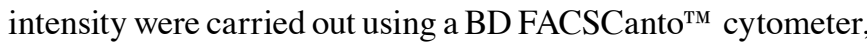
and the data were analyzed using the WinMDI 2.8 program (29). A total of $10^{5}$ cells was analyzed for each series.

Immunocytochemical, immunoblot and GJIC analyses. For the immunocytochemical analysis, the cells were fixed with methanol:acetone $\left(7: 3,-20^{\circ} \mathrm{C}\right)$, labelled with rabbit anti-Cx43 IgG (Sigma), counterstained with Alexa 488-conjugated goat anti-rabbit IgG (Molecular Probes) and $0.5 \mu \mathrm{g} / \mathrm{ml}$ bis-benzimide (Hoechst) and visualized using a Leica DM IRE2 microscope as presviously described (30). Similarly, immunoblot analysis was performed according to established protocols (31). Extracts from brown adipose tissue and liver were used as a PPAR $\alpha$-positive control. The intensity of GJIC was semi-quantitatively measured using a Leica DM IRE2 time-lapse system with some modifications (31). The results are expressed as a coupling ratio $\left(c_{r}\right)$, i.e. the number of positive recipient (identified using ImageJ free-ware) cells per one donor cell, and a coupling index $\left(\mathrm{c}_{\mathrm{i}}\right)$, defined as a percentage of coupled donor cells. At least 50 donor cells per Petri dish were analyzed in three independent experiments performed for each condition and analyzed using the Student's t-test.

Statistical analysis. Each parameter was calculated as the mean and standard error of the mean (SEM). The statistical significance was either determined by the Student's t-test with $\mathrm{p}<0.01$ considered to indicate significant differences or, where indicated, by the non-parametric Mann-Whitney test.

\section{Results}

Effect of fenofibrate on the morphology and motility of $D U-145$ cells. Cancer cell morphology and motility is crucial for cancer invasion (1). Analysis of the effect of fenofibrate on the morphology of DU-145 cells revealed the increased abundance of long and thin processes in the cells cultured with fenofibrate (Fig. 1B-D compared to A). This effect was correlated with a dose-dependent inhibition of 'contactstimulated' DU-145 cell motility (Fig. 1F-H compared to E). For example, the value of the average velocity of cell movement (AVCM) decreased from $50.8 \mu \mathrm{m} / \mathrm{h}$ in the control conditions to $14.1 \mu \mathrm{m} / \mathrm{h}$ in the DU-145 populations treated with $100 \mu \mathrm{M}$ fenofibrate (Fig. 1I; Table I). However, the effect of fenofibrate on DU-145 cell morphology and motility observed over a broad range of fenofibrate concentrations (25-100 $\mu \mathrm{M})$ did not affect DU-145 cell viability (Fig. 1J). This observation excludes the explanation that the inhibition of DU-145 cell motility was a result of compromised cell viability.

Fenofibrate inhibits the motility of DU-145 cells in a PPAR $\alpha$ independent manner. To elucidate the mechanisms involved in the inhibitory effect of fenofibrate on DU-145 cell motility, we investigated the role of $\operatorname{PPAR} \alpha$ receptors, plasma 

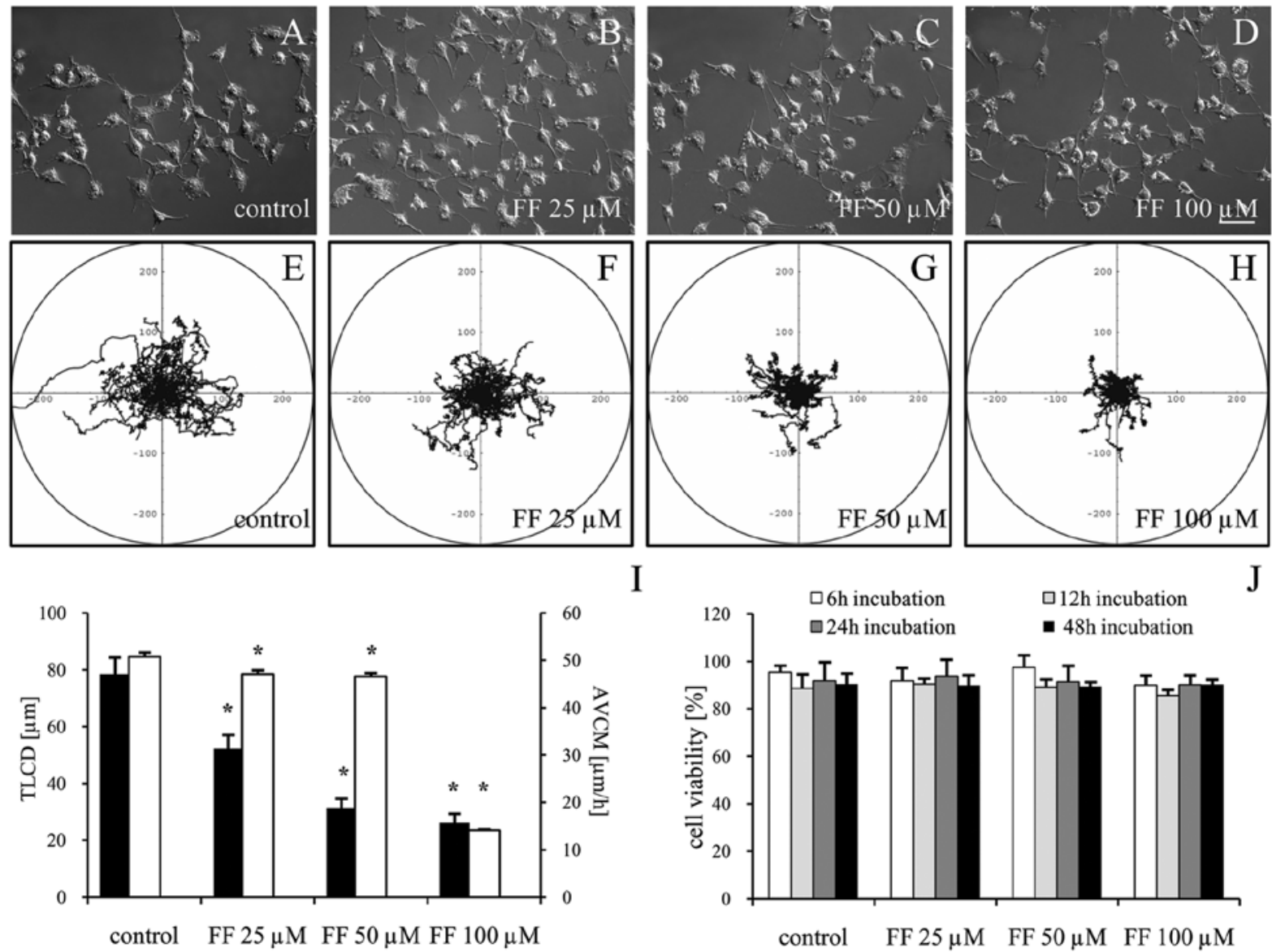

Figure 1. Dose-dependent effect of fenofibrate (FF) on the morphology (A-D) and motile activity (E-I) of DU-145 cells. Dose-dependent fenofibrate-induced changes in cell morphology correlated with an inhibition in DU-145 cell motility as shown by the circular diagrams depicting the trajectories of cells cultured under control conditions (A, E) and in the presence of $25 \mu \mathrm{M}(\mathrm{B}, \mathrm{F}), 50 \mu \mathrm{M}(\mathrm{C}, \mathrm{G})$ and $100 \mu \mathrm{M}(\mathrm{D}, \mathrm{H})$ fenofibrate (quantitatively summarized in I; also see Materials and methods) in a manner independent of DU-145 cell viability (J). Cell trajectories were registered $16 \mathrm{~h}$ after cell seeding and $15 \mathrm{~min}$ after the application of fenofibrate. Trajectories of DU-145 cells (registered for $7 \mathrm{~h}$ at 300 -sec time intervals) are presented in the form of circular diagrams (axis scale in $\mu \mathrm{m}$ ) drawn with the initial point of each trajectory placed at the origin of the plot. "Statistical significance vs. the relevant control at $\mathrm{p}<0.01$, using the MannWhitney test and Student's t-test for cell migration and morphology, respectively. Scale bar, $40 \mu \mathrm{m}$.

Table I. Effect of fenofibrate on the contact-stimulated motile activity of DU-145 cells.

\begin{tabular}{lrccc}
\hline Parameters & Control & Fenofibrate $(25 \mu \mathrm{M})$ & Fenofibrate $(50 \mu \mathrm{M})$ & Fenofibrate $(100 \mu \mathrm{M})$ \\
\hline Total length of cell trajectory $(\mu \mathrm{m})$ & $355.8 \pm 6.2$ & $329.9 \pm 5.7^{\mathrm{a}}$ & $326.2 \pm 4.9^{\mathrm{a}}$ & $98.8 \pm 1.9^{\mathrm{a}}$ \\
Average velocity of cell movement $(\mu \mathrm{m} / \mathrm{h})$ & $50.8 \pm 0.9$ & $47.1 \pm 0.8^{\mathrm{a}}$ & $46.6 \pm 0.7^{\mathrm{a}}$ & $14.1 \pm 0.3^{\mathrm{a}}$ \\
Total length of cell displacement $(\mu \mathrm{m})$ & $78.5 \pm 5.9$ & $52.3 \pm 4.8^{\mathrm{a}}$ & $31.2 \pm 3.5^{\mathrm{a}}$ & $26.2 \pm 3.2^{\mathrm{a}}$ \\
Average rate of cell displacement $(\mu \mathrm{m} / \mathrm{h})$ & $11.2 \pm 0.8$ & $7.5 \pm 0.7^{\mathrm{a}}$ & $4.4 \pm 0.5^{\mathrm{a}}$ & $3.7 \pm 0.4^{\mathrm{a}}$ \\
\hline
\end{tabular}

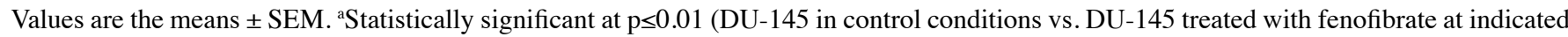
concentrations).

membrane fluidity $(19)$ and ROS metabolism $(10,17)$ in this process. Cultivation of DU-145 cells in the presence of $10 \mu \mathrm{M}$ GW9662, which effectively inhibits both PPAR $\gamma$ and PPAR $\alpha$ (32), did not restore the motility of DU-145 cells inhibited by fenofibrate (Fig. 2A). Thus, a PPAR-independent effect of fenofibrate on DU-145 cell motility is suggested. Furthermore, fluorescence anisotropy experiments revealed a lack of effect of fenofibrate on DU-145 cell membrane microviscosity
(Fig. 2B), indicating that fenofibrate inhibits DU-145 prostate cancer cell motility in a manner independent of the changes of DU-145 cell membrane properties. Instead, FACS analyses revealed increased levels of ROS in fenofibrate-treated DU-145 cells (Fig. 2C). Since ROS activity affects cancer cell motility and GJIC in cell populations (24), we further concentrated on the role of ROS in the regulation of these parameters. 
A
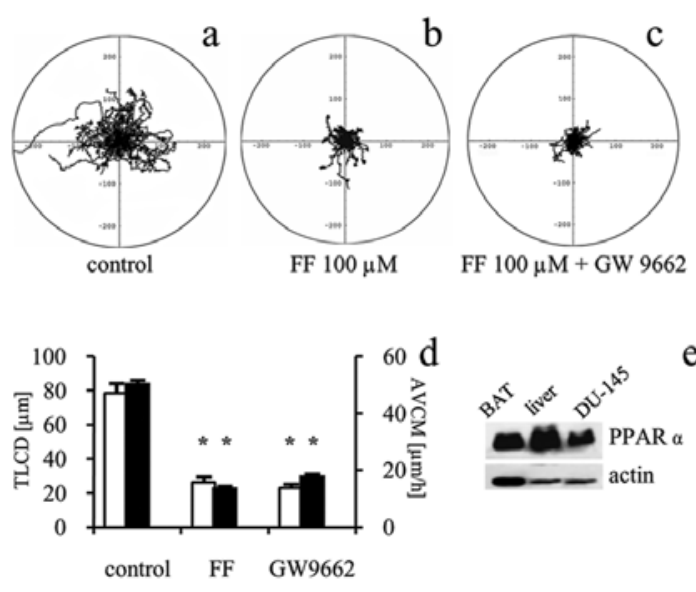

B

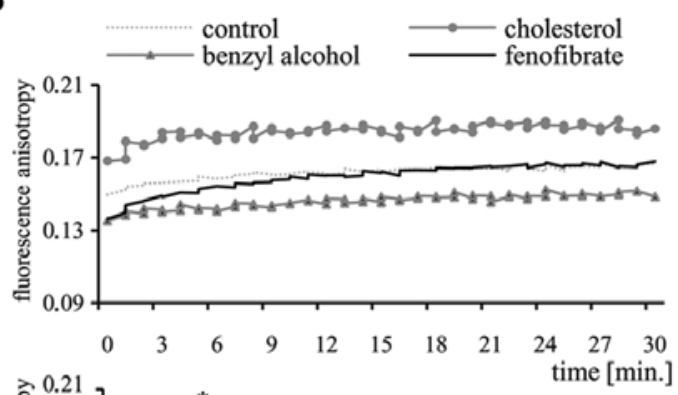

e

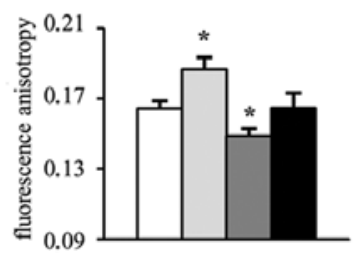

$\mathrm{C}$

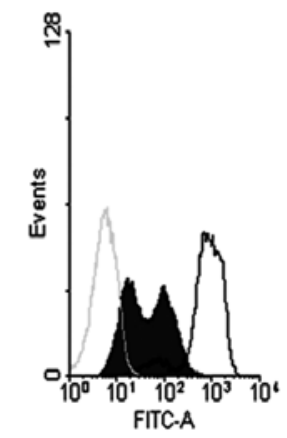

Figure 2. Mechanistic approach towards the identification of the signaling systems responsible for the inhibitory effect of fenofibrate on the motility of DU-145 cells addressing the possible involvement of PPAR $\alpha$ receptors (A), membrane microviscosity (B), and reactive oxygen species (C). (A) DU-145 cells express levels of PPAR $\alpha$ receptors comparable to the positive control [brown adipose tissue (BAT) and liver, e], yet, inhibition of their activity by GW9662 had no effect on the inhibitory effect of fenofibrate-induced DU-145 cell motility as shown in d (closed bars and open bars representing total length of cell displacement and average velocity of cell movement, respectively) and depicted in circular diagrams (a-c). (B) Similarly, no effect of fenofibrate on DU-145 cell membrane microviscosity was observed using dynamic (upper diagram) and static (lower diagram) analyses of fluorescence anisotropy, with cholesteroland benzyl alcohol-treated cells used as positive and negative control, respectively. (C) In contrast, a significant increase in ROS levels was observed in the fenofibrate-treated DU-145 cells [black line, compared to grey for pure medium (negative control) and black diagram as a positive control $\left(\mathrm{H}_{2} \mathrm{O}_{2}\right)$; for details see Materials and methods]. Trajectories of DU-145 cells (registered for $7 \mathrm{~h}$ at $300-\mathrm{sec}$ time intervals) are presented in the form of circular diagrams (axis scale in $\mu \mathrm{m}$ ) drawn with the initial point of each trajectory placed at the origin of the plot. "Statistical significance vs. the relevant control at $\mathrm{p}<0.01$, using the Mann-Whitney test and Student's t-test for cell migration and membrane microviscosity, respectively.
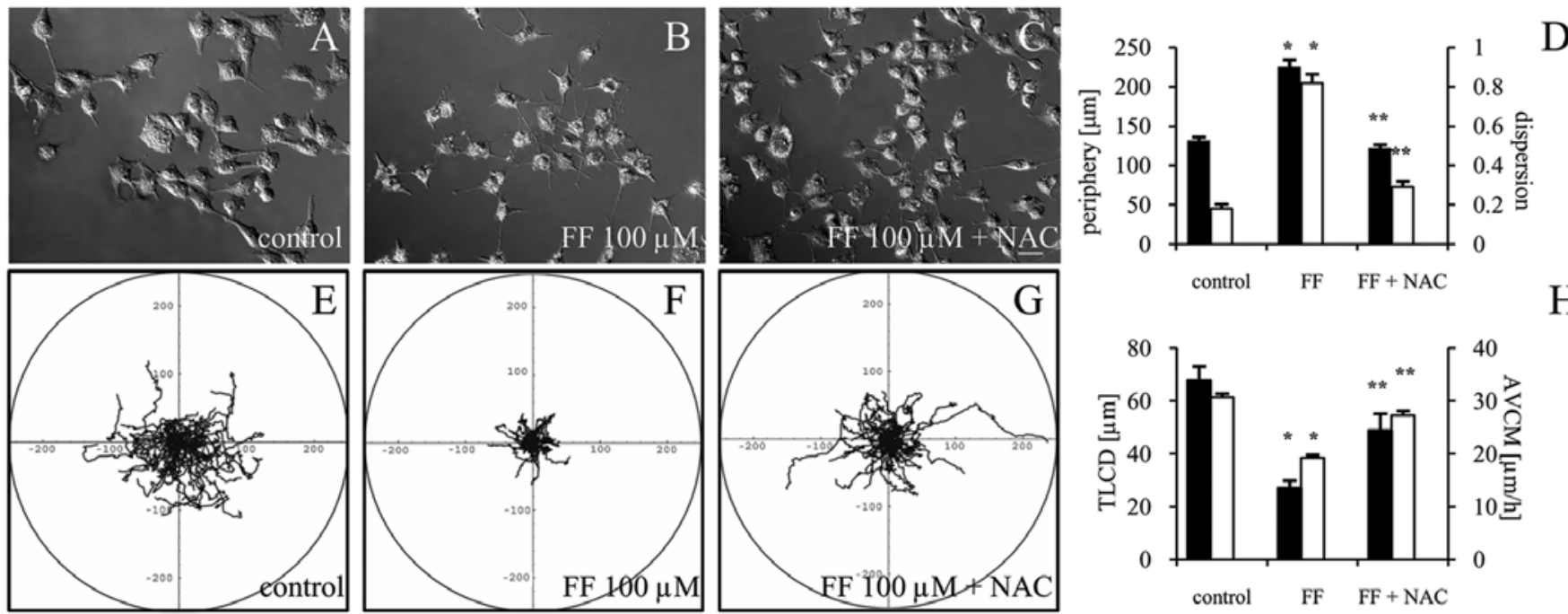

$\mathrm{H}$

Figure 3. Effect of N-acetyl-L-cysteine (NAC) on the morphology (A-D) and motility (E-H) of DU-145 cells maintained under fenofibrate-induced oxidative stress. Fenofibrate-induced changes in the average cell size and shape, visualized by the length of cell periphery and cell dispersion, respectively (B compared to A) were partially reversed by NAC as shown in C and quantitatively summarized in D (closed bars and open bars representing length of cell periphery and cell dispersion, respectively). Similarly, NAC partially restored fenofibrate-inhibited (F compared to E) DU-145 cell motility as illustrated in G and summarized in $\mathrm{H}$ (closed bars and open bars representing total length of cell displacement and average velocity of cell movement, respectively). Trajectories of DU-145 cells (registered for $7 \mathrm{~h}$ at 300 sec-time intervals after $4 \mathrm{~h}$ of incubation) are presented in the form of circular diagrams (axis scale in $\mu \mathrm{m}$ ) drawn with the initial point of each trajectory placed at the origin of the plot. "Statistical significance for cells in fenofibrate vs. cells in pure medium at $\mathrm{p}<0.01$, using the Mann-Whitney test and Student's t-test for cell migration and morphology, respectively. ${ }^{* *}$ Statistical significance for cells treated with fenofibrate and NAC vs. cells treated with fenofibrate at $\mathrm{p}<0.01$, using the Mann-Whitney test and the Student's t-test for cell migration and morphology, respectively. Scale bar, $40 \mu \mathrm{m}$.

$N$-acetyl-L-cysteine partially reverses the inhibitory effect of fenofibrate on the motility of DU-145 cells. N-acetyl-Lcysteine (NAC) is an effective scavenger of free radicals (24). Therefore, we further assessed the morphology (Fig. 3A-D) and motility (Fig. 3E-H) of DU-145 cells cultivated in the presence of fenofibrate and NAC. Increased lengths of the cell periphery and values of cell shape dispersion noted upon fenofibrate treatment (Fig. 3B compared to A) were diminished 
Table II. Parameters characterizing the motility of DU-145 cells under control conditions and in the presence of fenofibrate or fenofibrate and NAC.

\begin{tabular}{lcrr}
\hline Parameters & Control & Fenofibrate $(100 \mu \mathrm{M})$ & Fenofibrate $(100 \mu \mathrm{M})+\mathrm{NAC}$ \\
\hline Total length of cell trajectory $(\mu \mathrm{m})$ & $215.1 \pm 5.3$ & $135.2 \pm 3.4^{\mathrm{a}}$ & $191.5 \pm 5.4^{\mathrm{b}}$ \\
Average velocity of cell movement $(\mu \mathrm{m} / \mathrm{h})$ & $30.7 \pm 0.7$ & $19.3 \pm 0.5^{\mathrm{a}}$ & $27.3 \pm 0.8^{\mathrm{b}}$ \\
Total length of cell displacement $(\mu \mathrm{m})$ & $67.9 \pm 5.2$ & $27.2 \pm 2.7^{\mathrm{a}}$ & $49.0 \pm 6.1^{\mathrm{b}}$ \\
Average rate of cell displacement $(\mu \mathrm{m} / \mathrm{h})$ & $9.7 \pm 0.74$ & $3.8 \pm 0.4^{\mathrm{a}}$ & $7.0 \pm 0.9^{\mathrm{b}}$
\end{tabular}

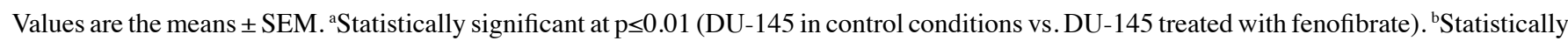
significant at $\mathrm{p} \leq 0.01$ (DU-145 in the presence of fenofibrate vs. DU-145 treated with fenofibrate and NAC).
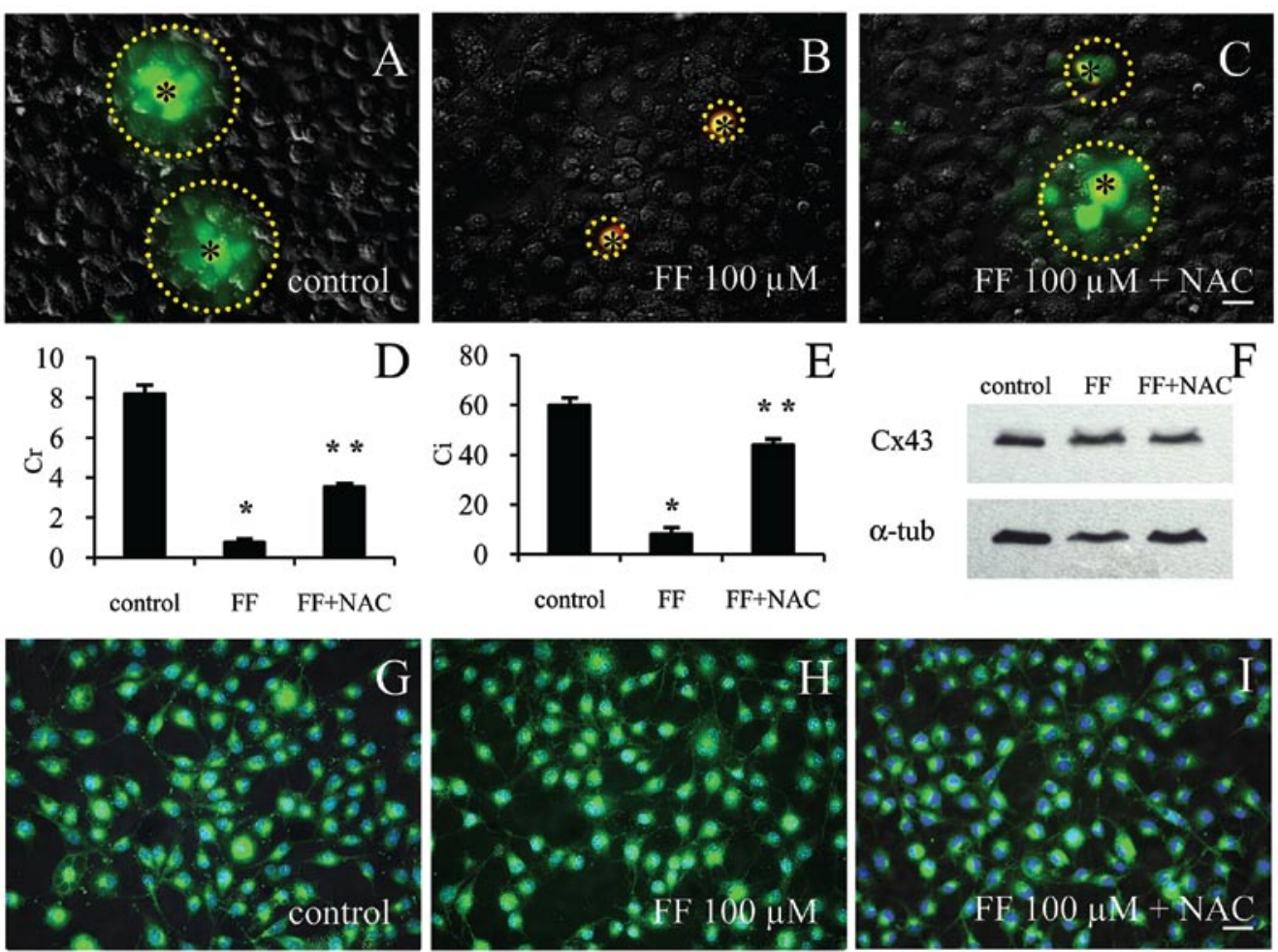

Figure 4. N-acetyl-L-cysteine (NAC) partially attenuates fenofibrate-induced inhibition of GJIC in DU-145 cell populations. A relatively intensive intercellular spreading of calcein (green) in DU-145 cells was observed under the control conditions (A), but was completely absent in the presence of $100 \mu \mathrm{M}$ fenofibrate (B), while parallel application of NAC partially restored GJIC in the analyzed cell populations as visualized in (C) and quantitatively summarized by semiquantitative analyses of the coupling ratio (D) and coupling index (E) (for details see Materials and methods). On the other hand, no effect of fenofibrate on Cx43 expression (F) was observed while a decreased abundance of Cx43-positive spots was observed in the fenofibrate-treated DU-145 cell populations when compared to the control variants (H compared to G), which was again restored by NAC treatment (I). Dotted circles in A-C show the range of calcein transfer between the donor (marked with *) and the calcein-positive recipient cells. (D-E) *Statistical significance for cells treated with fenofibrate vs. cells in pure medium at $\mathrm{p}<0.01$ (Student's t-test). ${ }^{* *}$ Statistical significance for cells treated with fenofibrate and NAC vs. cells treated with fenofibrate at $\mathrm{p}<0.01$ (Student's t-test). Scale bar, $40 \mu \mathrm{m}$.

in the presence of NAC (Fig. 3C compared to A; summarized in D). This effect was correlated with an NAC-induced reversal of the inhibitory effect of fenofibrate on DU-145 cell motility illustrated by quantitative analyses of cell translocations. For example, the average TLCD of the DU-145 cells decreased from $67.9 \mu \mathrm{m}$ (Fig. 3E, control) to $27.2 \mu \mathrm{m}$ in the presence of $100 \mu \mathrm{M}$ fenofibrate (Fig. 3F) but reached $49.0 \mu \mathrm{m}$ in the presence of both fenofibrate and NAC (Fig. 3G; summarized in $\mathrm{H}$ and Table II). These data indicate that fenofibrateinduced oxidative stress determines DU-145 cell invasive potential via the effect on their motility.
Effect of $N$-acetyl-L-cysteine on GJIC in fenofibrate-treated DU-145 cell populations. Since GJIC is a factor contributing to cancer cell invasion (23) and gap junctional channels are targets of ROS (24), we further assessed the influence of fenofibrate on GJIC in DU-145 cell populations and the role of ROS in this process. Fluorochrome transfer analyses revealed relatively high levels of GJIC in the DU-145 cell populations (Fig. 4A), which were attenuated by fenofibrate treatment (Fig. 4B). Notably, NAC partially restored GJIC in fenofibratetreated populations as illustrated in Fig. 4C (compared to B) and quantitatively summarized in Fig. 4D and E as estimated 
for cell populations cultured in the presence of fenofibrate alone or fenofibrate and NAC. Cx43 is the principal connexin determining GJIC in DU-145 cell populations. No significant differences in $\mathrm{Cx} 43$ expression levels were observed between the control and fenofibrate-treated cells (Fig. 4F). However, a decreased abundance of $\mathrm{Cx} 43$-positive plaques was noted in the fenofibrate-treated DU-145 cell populations (Fig. $4 \mathrm{H}$ ) when compared to the control variants (Fig. 4G). Similarly, NAC treatment increased the number of such plaques in fenofibrate-treated DU-145 cell populations (Fig. 4I). Thus, ROS may inhibit cancer progression via an effect on GJIC in cancer cell populations.

\section{Discussion}

Studies have suggested that fenofibrate influences cancer promotion and progression via the inhibitory effect on cell proliferation and the activation of apoptosis (14-16). Fenofibrate was also shown to attenuate the invasive potential of cancer cells (18). However, the involvement of fenofibrate in the regulation of prostate cancer cell properties crucial for their invasive potential, such as cell motility and the function of gap junctions, has not yet been studied. Here, we showed the effect of fenofibrate on the motility of human prostate DU-145 cells in the context of gap junctional coupling in prostate cancer cell populations.

Cell motility is a factor vital to the process of tumor invasion, and its 'contact-stimulated' variant may be particularly crucial for cancer cell dissemination $(26,27)$. Time-lapse experiments revealed that fenofibrate inhibited 'contactstimulated' motility of DU-145 cells. An inhibitory effect of fenofibrate on the invasiveness of melanoma $(12,13)$ and glioblastoma cell migration (18) has previously been demonstrated. These experiments, however, did not address the problem of 'contact-stimulated' cell motility and, moreover, described PPAR $\alpha$-dependent cell responses to fenofibrate. Here, we showed for the first time that the inhibitory effect of fenofibrate on 'contact-stimulated' DU-145 cell motility is independent of PPAR $\alpha$ activation.

Many PPAR ligands possess extra-receptor biological activities and fenofibrate is no exception (21). For instance, fenofibrate increases cell membrane rigidity imitating the action of cholesterol (19). Membrane microviscosity was also found to affect cell motility and the activity of cell membraneassociated growth factor receptors (33). Thus, alternative signaling pathways may be involved in the regulation of cancer cell migration by fenofibrate. However, neither significant changes in cell membrane microviscosity following fenofibrate treatment nor the effect of fenofibrate on DU-145 cell viability was observed in this study. The latter remains in contrast to the effect of fenofibrate found in melanoma and medulloblastoma cells $(13,34)$.

Instead, an increase in ROS accumulation in fenofibratetreated DU-145 cells was observed, a phenomenon previously suggested to modulate glioma cell motility in a manner dependent on the metabolic switch induced by PPAR $\alpha$ activation (18). Our findings revealing a partial restoration of fenofibrate-inhibited cell motility by NAC indicate that the inhibition of DU-145 cell motility relies on the effect(s) of fenofibrate on intracellular ROS levels. The mechanism of
PPAR $\alpha$-independent ROS accumulation by fenofibrate in DU-145 cells is unknown but may depend on physicochemical properties of fibrates. They allowed them to interact with various hydrophobic components of the mitochondrial electron respiratory chain leading to its disruption at the level of NADH cytochrome c reductase (11) and increased oxidative stress. Notably, the application of NAC restored both fenofibrate-inhibited DU-145 cell motility and GJIC in DU-145 cell populations.

Fibrates in general and fenofibrate in particular, are primarily 'vasoactive agents', yet are subsequently proposed to affect carcinogenesis (35). Our data broaden the spectrum of potential anti-cancer activities of fenofibrate, unrelated to those originally defined as primary targets, potentially resulting in new applications. In particular, we suggest that fenofibrate-induced ROS accumulation exerts an inhibitory effects on the function of $\mathrm{Cx} 43$ in DU-145 cell populations. A similar effect was previously demonstrated for normal HEK-293 cell populations, where GJIC inhibited by organotin compounds was partially restored by NAC (24). High levels of connexin expression and GJIC often correlate with a single cancer cell invasive potential indicating that connexins $(7,22)$ and gap junctional coupling $(8,23)$ are crucial for cancer progression. We suggest the existence of a common system which regulates cancer cell motility and gap junctional coupling in prostate cancer cell populations. It is involved in cancer progression $(36,37)$ and partially depends on reactive oxygen species. The interference of fenofibrate with this system sheds new light on possible fenofibrate applications in cancer prevention and prophylactics.

\section{Acknowledgements}

This research project was financed in part, by the European Union within the European Regional Development Fund under the Operational Programme Innovative Economy (UDA-POIG.01.03.01-14-036/09-00); and from the Polish Ministry of Scientific Research and Higher Education No. N N302 061936 and N N301 050236. The Faculty of Biochemistry, Biophysics and Biotechnology of the Jagiellonian University is a beneficiary of the structural funds from the European Union (grant no. POIG.02.01.00-12-064/08 'Molecular Biotechnology for Health' and POIG 01.02-00$109 / 99$ 'Innovative methods of stem cell application in medicine').

\section{References}

1. Friedl P and Wolf K: Plasticity of cell migration: a multiscale tuning model. J Exp Med 207: 11-19, 2010.

2. Yamasaki H, Krutovskikh V, Mesnil M, et al: Role of connexin (gap junction) genes in cell growth control and carcinogenesis. C R Acad Sci III 322: 151-159, 1999.

3. Laird DW: Life cycle of connexins in health and disease. Biochem J 394: 527-543, 2006.

4. Trosko JE: Gap junctional intercellular communication as a biological 'Rosetta stone' in understanding, in a systems biological manner, stem cell behavior, mechanisms of epigenetic toxicology, chemoprevention and chemotherapy. J Membr Biol 218: 93-100, 2007.

5. Fujimoto E, Satoh H, Negishi E, et al: Negative growth control of renal cell carcinoma cell by connexin 32: possible involvement of Her-2. Mol Carcinog 40: 135-142, 2004. 
6. Lin JH, Takano T, Cotrina ML, et al: Connexin 43 enhances the adhesivity and mediates the invasion of malignant glioma cells J Neurosci 22: 4302-4311, 2002.

7. Pollmann MA, Shao Q, Laird DW and Sandig M: Connexin 43 mediated gap junctional communication enhances breast tumor cell diapedesis in culture. Breast Cancer Res 7: R522-R534, 2005.

8. Cronier L, Crespin S, Strale PO, Defamie N and Mesnil M: Gap junctions and cancer: new functions for an old story. Antioxid Redox Signal 11: 323-338, 2009.

9. Chinetti G, Fruchart JC and Staels B: Peroxisome proliferatoractivated receptors (PPARs): nuclear receptors at the crossroads between lipid metabolism and inflammation. Inflamm Res 49: 497-505, 2000

10. Jiao HL and Zhao BL: Cytotoxic effect of peroxisome proliferator fenofibrate on human HepG2 hepatoma cell line and relevant mechanisms. Toxicol Appl Pharmacol 185: 172-179, 2002.

11. Scatena R, Bottoni P, Vincenzoni F, et al: Bezafibrate induces a mitochondrial derangement in human cell lines: a PPARindependent mechanism for a peroxisome proliferator. Chem Res Toxicol 16: 1440-1447, 2003.

12. Grabacka M, Placha W, Plonka PM, et al: Inhibition of melanoma metastases by fenofibrate. Arch Dermatol Res 296: 54-58, 2004.

13. Grabacka M, Plonka PM, Urbanska K and Reiss K: Peroxisome proliferator-activated receptor alpha activation decreases metastatic potential of melanoma cells in vitro via down-regulation of Akt. Clin Cancer Res 12: 3028-3036, 2006.

14. Panigrahy D, Kaipainen A, Huang S, et al: PPARalpha agonist fenofibrate suppresses tumor growth through direct and indirect angiogenesis inhibition. Proc Natl Acad Sci USA 105: 985-990, 2008 .

15. Thuillier P, Anchiraico GJ, Nickel KP, et al: Activators of peroxisome proliferator-activated receptor-alpha partially inhibit mouse skin tumor promotion. Mol Carcinog 29: 134-142, 2000.

16. Saidi SA, Holland CM, Charnock-Jones DS and Smith SK In vitro and in vivo effects of the PPAR-alpha agonists fenofibrate and retinoic acid in endometrial cancer. Mol Cancer 5: 13, 2006.

17. Grabacka M and Reis K: Effects on cellular metabolism and inflammation. PPAR Res 2008: 930705, 2008.

18. Drukala J, Urbanska K, Wilk A, et al: ROS accumulation and IGF-IR inhibition contribute to fenofibrate/PPARalpha-mediated inhibition of glioma cell motility in vitro. Mol Cancer 9: 159, 2010.

19. Gamerdinger M, Clement AB and Behl C: Cholesterol-like effects of selective cyclooxygenase inhibitors and fibrates on cellular membranes and amyloid-beta production. Mol Pharmacol 72: 141-151, 2007.

20. Scatena R, Martorana GE, Bottoni P and Giardina B: Mitochondrial dysfunction by synthetic ligands of peroxisome proliferator activated receptors (PPARs). IUBMB Life 56 : 477-482, 2004.

21. Scatena R, Bottoni P and Giardina B: Mitochondria, PPARs, and cancer: Is receptor-independent action of PPAR agonists a key? PPAR Res 2008: 256251, 2008.
22. Miekus K, Czernik M, Sroka J, Czyz J and Madeja Z: Contact stimulation of prostate cancer cell migration: the role of gap junctional coupling and migration stimulated by heterotypic cell-to-cell contacts in determination of the metastatic phenotype of Dunning rat prostate cancer cells. Biol Cell 97: 893-903, 2005.

23. Czyz J: The stage-specific function of gap junctions during tumourigenesis. Cell Mol Biol Lett 13: 92-102, 2008.

24. Sroka J, Czyz J, Wojewoda M and Madeja Z: The inhibitory effect of diphenyltin on gap junctional intercellular communication in HEK-293 cells is reduced by thioredoxin reductase 1 . Toxicol Lett 183: 45-51, 2008.

25. Sroka J, Antosik A, Czyz J, et al: Overexpression of thioredoxin reductase 1 inhibits migration of HEK-293 cells. Biol Cell 99: 677-687, 2007.

26. Madeja Z, Miekus K, Sroka J, Djamgoz MB and Korohoda W: Homotypic cell-cell contacts stimulate the motile activity of rat prostate cancer cells. BJU Int 88: 776-786, 2001.

27. Madeja Z, Szymkiewicz I, Zaczek A, et al: Contact-activated migration of melanoma B16 and sarcoma XC cells. Biochem Cell Biol 79: 425-440, 2001.

28. Butkerait P, Zheng Y, Hallak H, et al: Expression of the human 5-hydroxytryptaminel A receptor in Sf9 cells. Reconstitution of a coupled phenotype by co-expression of mammalian $\mathrm{G}$ protein subunits. J Biol Chem 270: 18691-18699, 1995.

29. Miekus K, Jarocha D, Trzyna E and Majka M: Role of I-TACbinding receptors CXCR3 and CXCR7 in proliferation, activation of intracellular signaling pathways and migration of various tumor cell lines. Folia Histochem Cytobiol 48: 104-111, 2010

30. Czyz J, Guan K, Zeng Q and Wobus AM: Loss of betal integrin function results in upregulation of connexin expression in embryonic stem cell-derived cardiomyocytes. Int J Dev Biol 49: 33-41, 2005.

31. Daniel-Wojcik A, Misztal K, Bechyne I, et al: Cell motility affects the intensity of gap junctional coupling in prostate carcinoma and melanoma cell populations. Int J Oncol 33: 309-315, 2008.

32. Leesnitzer LM, Parks DJ, Bledsoe RK, et al: Functional consequences of cysteine modification in the ligand binding sites of peroxisome proliferator activated receptors by GW9662. Biochemistry 41: 6640-6650, 2002.

33. Ghosh PK, Vasanji A, Murugesan G, et al: Membrane microviscosity regulates endothelial cell motility. Nat Cell Biol 4 : 894-900, 2002.

34. Urbanska K, Pannizzo P, Grabacka M, et al: Activation of PPARalpha inhibits IGF-I-mediated growth and survival responses in medulloblastoma cell lines. Int J Cancer 123 1015-1024, 2008.

35. Dellavalle RP, Nicholas MK and Schilling LM: Melanoma chemoprevention: a role for statins or fibrates? Am J Ther 10: 203-210, 2003.

36. Czernik M, Sroka J, Madeja Z and Czyz J: Apigenin inhibits growth and motility but increases gap junctional coupling intensity in rat prostate carcinoma (MAT-LyLu) cell populations. Cell Mol Biol Lett 13: 327-338, 2008.

37. Miekus K and Madeja Z: Genistein inhibits the contact-stimulated migration of prostate cancer cells. Cell Mol Biol Lett 12: 348-361, 2007 